\title{
Comments
}

\section{Absence of Crystalline Ordering in Two Dimensions}

\author{
Jürg Fröhlich ${ }^{1}$ and Charles-Edouard Pfister ${ }^{2}$ \\ 1 Theoretische Physik, ETH-Hönggerberg, CH-8093 Zürich, Switzerland \\ 2 Département de mathématiques, E.P.F.-L, CH-1015 Lausanne, Switzerland
}

\begin{abstract}
We give conditions on the potential of a classical particle system, which imply absence of crystalline ordering in two dimensions. We thereby correct and extent some results in a previous paper.
\end{abstract}

\section{Introduction}

In an earlier paper [1] we gave a modified version of Mermin's argument for the absence of crystalline ordering in two-dimensional classical systems of point particles, [2]. In this note we would like to clarify and correct our discussion in [1], following Theorem 1 in that paper, and describe the kind of potentials for which our results apply. Since this note is a complement to [1], we use the same notations as in [1], and we do not repeat the basic definitions.

\section{Relative Entropy Argument}

We consider a system of point particles in $\mathbb{R}^{2}$. The configurations of the system are identified with the subsets, $\omega$, of $\mathbb{R}^{2}$ which are locally finite: $x \in \omega$ means that there is a particle at $x$, and, for any bounded set $\Lambda, \omega_{\Lambda}=\omega \cap \Lambda$ is a finite subset of $\mathbb{R}^{2}$. The interaction is given by a two-body translation invariant potential $\phi: \mathbb{R}^{2} \rightarrow \mathbb{R}$, $\phi(x)=\phi(-x)$, and we suppose that
A) $\phi$ is stable and regular;
B) $\phi$ is of class $C^{2}$, except at the origin.

The energy of a particle at $x$ in the configuration $\omega$ is

$$
H_{\phi}(x \mid \omega) \equiv H(x \mid \omega)=\sum_{\substack{y: x \neq y \\ y \in \omega}} \phi(x-y),
$$

J. Fröhlich: Comment on "Absence of Crystalline Ordering in two Dimensions" 
and the energy of $n$ particles at $x_{1}, \ldots, x_{n}$ is denoted by $U\left(x_{1}, \ldots, x_{n}\right)$. Let $P$ be a Gibbs state (equilibrium state) for an activity $z$ and inverse temperature $\beta$. We introduce the $n$-point correlation function

$$
\varrho_{P}\left(x_{1}, \ldots, x_{n}\right)=z^{n} e^{-\beta U\left(x_{1}, \ldots, x_{n}\right)}\left\langle\prod_{i=1}^{n} e^{-\beta H\left(x_{i} \mid \omega\right)}\right\rangle_{P}
$$

(see [3], Chap. 4, and [4]).

We choose a fixed, extremal Gibbs state, $P$. Let $T_{a}$ represent the translation, $x \rightarrow x+a$, in $\mathbb{R}^{2}$. We propose to prove that $P=P_{a}$, with $P_{a} \equiv T_{a}^{-1} P$. To show this, we construct a sequence of states, $P_{n}, n \in \mathbb{N}, P_{n}=T_{n}^{-1} P$, where $T_{n}$ is a smooth bijective transformation $\mathbb{R}^{2} \rightarrow \mathbb{R}^{2}$, which coincides with $T_{a}$ on $\Lambda_{n}=\left\{x \in \mathbb{R}^{2}:|x| \leqq n\right\}$, and which is the identity transformation outside some bounded region $\Lambda$ (see [1], p. 284). We choose any fixed number $\varepsilon$ in $(0,1)$ and a non-negative smooth function $u$ on $\mathbb{R}^{+}$, which is monotone decreasing and has the properties

$$
u(x)=1 \text { if } x \leqq 1,\left|\frac{d u}{d x}\right| \leqq \varepsilon, u(x)=0 \text { if } x \geqq 2+1 / \varepsilon
$$

We define

$$
T_{n}: x \rightarrow x+a \cdot u\left(\frac{|x|}{n}\right), n \geqq 1 .
$$

If we can find an upper bound, uniformly in $n$, for $S\left(P_{n} \mid P\right)$, the relative entropy of $P_{n}$ with respect to $P$, then $P_{a}=P$. Technically it is easier to estimate $S\left(P_{n} \mid P\right)+S\left(P_{-n} \mid P\right)$, where $P_{-n}=T_{-n}^{-1} P$, and $T_{-n}$ is given by the same formula as $T_{n}$, with a replaced by $-a$. Thus we must find a constant $K$, independent of $n$, such that

$$
0 \leqq S\left(P_{n} \mid P\right)+S\left(P_{-n} \mid P\right) \leqq K<\infty
$$

The transformation $T_{n}$ is local, and this implies that $P_{n}$ is absolutely continuous with respect to $P$, with density

$$
\frac{d P_{n}}{d P}(\omega)=\left(\prod_{x \in \omega_{\Lambda}} J_{T_{n}}(x)\right) \exp \beta\left(H_{\Lambda}\left(\omega_{\Lambda} \mid \omega\right)-H_{\Lambda}\left(T_{n} \omega_{\Lambda} \mid \omega\right)\right) ;
$$

$\left(J_{T_{n}}(x)\right.$ is the Jacobian $(\geqq 0)$ of $T_{n}$, and $H_{\Lambda}\left(\omega_{\Lambda} \mid \omega\right)$ is the energy of the particles in $\Lambda$, taking into account their interactions with the particles outside $\Lambda$ ). This identity permits us to estimate (2.3); (see [1]):

$$
\begin{aligned}
S\left(P_{n} \mid P\right)+S\left(P_{-n} \mid P\right) & =-\left\langle\log \frac{d P_{n}}{d P}(\omega)+\log \frac{d P_{-n}}{d P}(\omega)\right\rangle_{P} \\
& \leqq 0\left(\frac{\varepsilon^{2}}{n^{2}}\right)\left(\left\langle N_{\Lambda}(\omega)\right\rangle_{P}+\left\langle\sum_{x \in \omega_{\Lambda}} \sum_{\substack{y \in \omega \\
x \neq y}} \Psi_{\varepsilon}(x-y)\right\rangle_{P}\right) .
\end{aligned}
$$

The first term comes from the Jacobians, $J_{T_{n}} ; N_{\Lambda}(\omega)$ counts the number of particles in $\Lambda$. In the second term, $\Psi_{\varepsilon}(x): \mathbb{R}^{2} \rightarrow \mathbb{R}$ is defined by ( $\varepsilon$ is the number used in the 
definition of $T_{n}$ )

$$
\Psi_{\varepsilon}(x)=\sup _{\substack{a \in \mathbb{R}^{2} \\|a|=1}} \sup _{\substack{t \in \mathbb{R} \\|t| \leqq \varepsilon|x|}}\left|\frac{d^{2}}{d t^{2}} \Phi(x+t a)\right||x|^{2} .
$$

The expression on the right side of (2.5) is given by

$$
0\left(\frac{\varepsilon^{2}}{n^{2}}\right)\left(\int_{\Lambda} d x \varrho_{P}(x)+\int_{\Lambda} d x \int_{\mathbb{R}^{2}} d y \varrho_{P}(x, y) \Psi_{\varepsilon}(x-y)\right) .
$$

Theorem. Let $\Phi$ be a translation invariant potential satisfying conditions $A$ and $B$. Let $P$ an extremal Gibbs state such that $\varrho_{P}(x)$ and $\varrho_{P}(x, y)$ are well-defined, and let $\Psi_{\varepsilon}(x)$ be given by (2.6). If there exist two finite constants $C_{1}$ and $C_{2}$ such that, for all bounded $\Lambda \subseteq \mathbb{R}^{2}$,

$$
\int_{\Lambda} d x \varrho_{P}(x)=\left\langle N_{\Lambda}(\omega)\right\rangle_{P} \leqq C_{1}|\Lambda|
$$

and

$$
\int_{\Lambda} d x \int_{\mathbb{R}^{2}} d y \varrho_{P}(x, y) \Psi_{\varepsilon}(x-y) \leqq C_{2}|\Lambda|
$$

$(|\Lambda|=$ area of $\Lambda)$, then $P$ is translation invariant.

Remarks.

1) The theorem is an immediate consequence of (2.7), since, in this expression, $|\Lambda|=0\left(n^{2}\right)$, and therefore (2.3) follows.

2) The theorem is equivalent to Theorem $1 \mathrm{in}$ [1]. Indeed, for any bounded $\Lambda^{\prime}$,

$$
\int_{\Lambda^{\prime}} d y \varrho_{P}(x, y) \Psi_{\varepsilon}(x-y)=z\left\langle H_{\Psi_{\varepsilon}}\left(x \mid \omega_{\Lambda^{\prime}}\right) e^{-\beta H(x \mid \omega)}\right\rangle_{P} .
$$

The proof of (2.8) is quite similar to the proof of Lemma 2.3 in [1]. The equivalence follows then by the monotone convergence theorem.

We now suppose that $P$ is a Gibbs state for which all correlation functions are well-defined, and that there exists a $\xi$ such that, for all $n$,

$$
\varrho_{P}\left(x_{1}, \ldots, x_{n}\right) \leqq \xi^{n} .
$$

Let a: $\mathbb{R}^{2} \rightarrow \mathbb{R}^{+}$. For any bounded subset $\Lambda$,

$$
\begin{aligned}
& \left\langle\exp \left(\sum_{x \in \omega_{A}} \alpha(x)\right)\right\rangle_{P} \\
& =1+\sum_{n \geqq 1} \frac{1}{n !} \int_{A} d x_{1} \ldots \int_{A} d x_{n} \varrho_{P}\left(x_{1}, \ldots, x_{n}\right) \prod_{i=1}^{n}\left(e^{\alpha\left(x_{i}\right)}-1\right) \\
& \leqq 1+\sum_{n \geqq 1} \frac{\xi^{n}}{n !}\left(\int_{\Lambda} d x\left(e^{\alpha(x)}-1\right)\right)^{n}=\exp \left(\xi \int_{\Lambda} d x\left(e^{\alpha(x)}-1\right)\right) .
\end{aligned}
$$

The proof of (2.10) is accomplished by writing

$$
\prod_{i=1}^{n} e^{\alpha\left(x_{i}\right)}=\prod_{i=1}^{n}\left(\left(e^{\alpha\left(x_{i}\right)}-1\right)+1\right)=\prod_{i=1}^{n}\left(f\left(x_{i}\right)+1\right)=\sum_{Y \subset\left\{x_{1}, \ldots, x_{n}\right\}} f_{Y},
$$


where $f(x) \equiv e^{\alpha(x)}-1, f_{\phi} \equiv 1, f_{Y} \equiv \prod_{x \in Y} f(x)$. Using (2.10) and the regularity of $\Phi$, we get

$$
\left\langle\prod_{i=1}^{2} e^{-\beta H\left(x_{i} \mid \omega_{\Lambda}\right)}\right\rangle_{P} \leqq \prod_{i=1}^{2}\left\langle e^{-2 \beta H\left(x_{i} \mid \omega_{\Lambda}\right)}\right\rangle_{P}^{1 / 2} \leqq \exp \left(\xi \int_{\mathbb{R}^{2}} d x\left(e^{2 \beta\left|\Phi_{-}(x)\right|}-1\right)\right)<\infty,
$$

where $\Phi_{-}(x)$ is the negative part of the potential $\Phi$. Thus, the hypotheses of the theorem are satisfied if

$$
\int_{\mathbb{R}^{2}} d x \Psi_{\varepsilon}(x) e^{-\beta \Phi(x)} \leqq C<\infty
$$

(see (2.2), (2.7), (2.12)).

Corollary. Let $\Phi$ be a potential satisfying the hypotheses of the theorem, and suppose that $P$ is an extremal Gibbs state with correlation functions satisfying

$$
\varrho_{P}\left(x_{1}, \ldots, x_{n}\right) \leqq \xi^{n}, \forall n
$$

If

$$
\int_{\mathbb{R}^{2}} d x \Psi_{\varepsilon}(x) e^{-\beta \Phi(x)}<\infty,
$$

then $P$ is translation invariant (in two dimensions).

Remarks.

1) The condition (2.13) is essentially a condition of integrability of $\Psi_{\varepsilon}$ at infinity. Indeed, for large $|x|, \exp (-\beta \Phi(x))$ is almost one and, for small $|x|$, the divergence which may appear in $\Psi_{\varepsilon}(x)$ is in general compensated by $\exp (-\beta \Phi(x))$. Hence, for a potential $\Phi(x)=\Phi(|x|)$, the main condition on $\Phi$ in the corollary is roughly speaking the integrability of $\Phi^{\prime \prime}(|x|)|x|^{2}$, for large $|x|$.

2) The conditions on the correlation functions are satisfied by the equilibrium states, whose existence has been proven by Ruelle, [4]. Therefore if $\Phi$ satisfies the hypotheses of the corollary, is superstable, and if $P$ is a tempered Gibbs state, then $P$ is translation invariant.

3) The above corollary replaces the corollary on p. 282 in [1].

Acknowledgements. We thank G. Benfatto and E. Olivieri for very helpful discussions.

\section{References}

1. Fröhlich, J., Pfister, C.-E.: On the absence of spontaneous symmetry breaking and of crystalline ordering in two-dimensional systems, Commun. Math. Phys. 81, 277-298 (1981)

2. Mermin, N.D.: Crystalline order in two dimensions. Phys. Rev. 176, 250-254 (1968)

3. Ruelle, D.: Statistical mechanics. New York: Benjamin 1969

4. Ruelle, D.: Superstable interactions in classical statistical mechanics. Commun. Math.Phys. 18, $127-159(1970)$

Communicated by A. Jaffe

Received December 23, 1985 